\title{
Photocurrent enhancement by spontaneous formation of a p-n junction in Ca-doped bismuth vanadate photoelectrodes
}

\author{
Fatwa F. Abdi, ${ }^{*[a]}$ David E. Starr, ${ }^{[a]}$ Ibbi Y. Ahmet ${ }^{[a]}$ and Roel van de Krol ${ }^{[a]}$
}

\begin{abstract}
The application of bismuth vanadate $\left(\mathrm{BiVO}_{4}\right)$ photoelectrodes for solar water splitting is hindered by the poor carrier transport. To overcome this, multiple donor doping strategies (dual doping, gradient doping, etc.) have been explored. Here, we show for the first time a successful introduction of calcium $(\mathrm{Ca})$ as an acceptor-type dopant into $\mathrm{BiVO}_{4}$ photoelectrodes. Interestingly, instead of obtaining cathodic photocurrents, the Ca-doped $\mathrm{BiVO}_{4}$ photoelectrodes show anodic photocurrents with an enhanced carrier separation efficiency. Through hard X-ray photoelectron spectroscopy (HAXPES), we show that this enhancement is caused by out-diffusion of $\mathrm{Ca}$ during the deposition process, which spontaneously creates a p-n junction within the $\mathrm{BiVO}_{4}$ layer. Overall, a significant two-fold improvement of the AM1.5 photocurrent is obtained upon Ca-doping. This study highlights the importance of controlled doping beyond a simple modulation of carrier concentration, but more importantly in enabling new device architectures in photoelectrode materials.
\end{abstract}

\section{Introduction}

In solar water splitting, metal oxides have emerged as potential candidates for photoelectrode materials, ${ }^{[1]}$ due to their general stability in aqueous solutions and low-cost. Among the various oxides, bismuth vanadate $\left(\mathrm{BiVO}_{4}\right)$ is one of the most promising; its reported photocurrent for water oxidation has rapidly increased from several tens of $\mu \mathrm{A} / \mathrm{cm}^{2}$ to larger than $5 \mathrm{~mA} / \mathrm{cm}^{2}$ (i.e., 2 orders of magnitude) within only a few years of development. ${ }^{[2]}$ This success has been achieved by focused efforts on identifying the main limiting factors of the material and systematically addressing them. Recombination at the $\mathrm{BiVO}_{4} /$ electrolyte interface was one of the main limitations, ${ }^{[3]}$ which has been overcome by surface modification with various co-catalysts and surface treatments. ${ }^{[4]}$ Another limitation of the material is poor carrier transport. ${ }^{[5]} \mathrm{BiVO}_{4}$ has a relatively low conductivity, as reflected in the low carrier mobility value of $\sim 10^{-2}$ $\mathrm{cm}^{2} / \mathrm{Ns}^{[5 \mathrm{a}, 6]}$ To mitigate this problem, efforts have been directed towards donor-type doping, i.e., compensating the low mobility with increased carrier concentration. ${ }^{[2 a, 7]}$ Tungsten (W) and molybdenum (Mo) are among the most effective dopants, with high AM1.5 photocurrents reported for either W- or Mo-doped $\mathrm{BiVO}_{4}$ photoanodes. ${ }^{[4 \mathrm{c}, 8]} \mathrm{Co}$-doping with e.g. $\mathrm{Mo} / \mathrm{W},{ }^{[9]} \mathrm{Fe} / \mathrm{Mo},{ }^{[10]}$

[a] Dr. F. F. Abdi*, Dr. D. E. Starr, Dr. I. Y. Ahmet, Prof. R. van de Krol Institute for Solar Fuels

Helmholtz-Zentrum Berlin für Materialien und $\mathrm{GmbH}$

Hahn-Meitner-Platz 1, 14109 Berlin, Germany

*E-mail: fatwa.abdi@helmholtz-berlin.de

Supporting information for this article is given via a link at the end of the document.
$\mathrm{Mo} / \mathrm{H}^{[11]}$ and $\mathrm{Fe} / \mathrm{W}^{[12]}$ has also resulted in enhanced photocurrents, although the exact nature of the synergistic effect of the co-dopants seems not yet fully understood. One issue with the high donor concentration in these photoanodes is that it also results in very narrow space charge regions and, therefore, poor charge separation efficiencies. To address this, we proposed a gradient doping strategy that distributes the electric field over a larger region of the sample. ${ }^{[8 d, 13]}$ While this indeed enhances the separation efficiency, the reported photocurrents for dense thin film $\mathrm{BiVO}_{4}$ photoelectrodes still fall short of the theoretical value of $7.3 \mathrm{~mA} / \mathrm{cm}^{2}$ (based on the $2.4 \mathrm{eV}$ bandgap of $\mathrm{BiVO}_{4}$ ).

Despite numerous efforts on donor doping of $\mathrm{BiVO}_{4}$, there are very few reports on acceptor-doped $\mathrm{BiVO}_{4}$ photoelectrodes. ${ }^{[14]}$ This is not surprising, since it seems counter-intuitive to introduce an acceptor dopant into an n-type semiconductor such as $\mathrm{BiVO}_{4}$. However, the successful introduction of an acceptortype dopant would enable the fabrication of $\mathrm{p}$-type $\mathrm{BiVO}_{4}$. This would allow the fabrication of $\mathrm{p}-\mathrm{n}$ or $\mathrm{p}-\mathrm{i}-\mathrm{n}$ homojunctions based on $\mathrm{BiVO}_{4}$. The advantage of this type of architecture is that it further extends the width of the space charge region (i.e., to both sides of the junction) and that it offers a convenient route to fabricate completely buried junctions.

The possibility of acceptor doping of $\mathrm{BiVO}_{4}$ has been explored in a density functional theory (DFT) study by Yin et al. ${ }^{[14 a]}$ Their calculations reveal that introducing strontium $(\mathrm{Sr})$ or calcium (Ca) on Bi-sites leads to the formation of very shallow acceptor levels with low defect formation energies. $\mathrm{Ca}^{2+}$ and $\mathrm{Sr}^{2+}$ have similar ionic radii $\left(1.12 \AA\right.$ and $1.26 \AA$ ) as $\mathrm{Bi}^{3+}(1.17 \AA),{ }^{[15]}$ which likely explains the low defect formation energies. This is also consistent with the observation that solid solutions of $\mathrm{CaMoO}_{4}-$ $\mathrm{BiVO}_{4}$ and $\mathrm{CaWO}_{4}-\mathrm{BiVO}_{4}$ are easily formed. ${ }^{[16]}$ In this manuscript, we successfully introduce $\mathrm{Ca}$ as an acceptor-dopant into spray-pyrolysed $\mathrm{BiVO}_{4}$ films, ${ }^{[17]}$ consistent with the prediction from DFT. ${ }^{[14 a]}$ Interestingly, cathodic photocurrents expected from an acceptor-doped $\mathrm{BiVO}_{4}$ was not observed. Instead, we show that the carrier separation and injection efficiencies in the Ca-doped $\mathrm{BiVO}_{4}$ films are enhanced through the formation of a gradient $\mathrm{p}-\mathrm{n}$ homojunction within the film. This homojunction is formed through spontaneous out-diffusion of $\mathrm{Ca}$ towards the surface of the film, as evidenced by hard X-ray photoelectron spectroscopy (HAXPES) measurements. While $p$ $n$ heterojunctions based on $\mathrm{BiVO}_{4}$ (e.g., p-BiOl/n-BiVO ${ }_{4}$ and $\mathrm{p}$ $\mathrm{Co}_{3} \mathrm{O}_{4} / \mathrm{n}-\mathrm{BiVO}_{4}$ ) have been reported, ${ }^{[18]}$ to the best of our knowledge this is the first report on a $\mathrm{BiVO}_{4} \mathrm{p}$-n homojunction.

\section{Results and Discussion}


Spray deposited Ca-doped $\mathrm{BiVO}_{4}$ films were made by adding calcium nitrate $\left(\mathrm{Ca}\left(\mathrm{NO}_{3}\right)_{2} \cdot 4 \mathrm{H}_{2} \mathrm{O}\right)$ to the $\mathrm{BiVO}_{4}$ precursor solution (see Experimental section). To ensure that the introduction of the dopant is electronically compensated with holes, we codoped the $\mathrm{BiVO}_{4}$ with excess vanadium, so that the $1: 1$ stoichiometry of $(\mathrm{Ca}+\mathrm{Bi}): \mathrm{V}$ is preserved. The dopant introduction reaction can be written as follows in the usual Kröger-Vink notation:

$$
2 \mathrm{CaO}+\mathrm{V}_{2} \mathrm{O}_{5}+\frac{1}{2} \mathrm{O}_{2}(\mathrm{~g}) \stackrel{2 \mathrm{BiVO}_{4}}{\longrightarrow} 2 \mathrm{Ca}_{\mathrm{Bi}}^{\prime}+2 \mathrm{~V}_{\mathrm{V}}^{\mathrm{x}}+8 \mathrm{O}_{\mathrm{O}}^{\mathrm{x}}+2 \mathrm{~h}^{\bullet}
$$

Figure 1a shows a photograph of an undoped and a $1 \% \mathrm{Ca}$ doped $\mathrm{BiVO}_{4}$ film. No significant differences in the visual appearance of the films are observed. Scanning electron micrographs (SEM) of both undoped and $1 \%$ Ca-doped $\mathrm{BiVO}_{4}$ (Fig. $1 \mathrm{~b}$ and c) reveal similar film morphologies. The features of the Ca-doped films look slightly less smooth, but the feature size is approximately the same for both films $(\sim 100-200 \mathrm{~nm})$. Crosssection SEM images also show similar thickness $(\sim 100 \mathrm{~nm})$ for both films (Fig. S1, Supplementary Information). The UV-vis absorption spectra of both films are also similar, as shown in Figure S2 (Supplementary Information). The Ca-doped $\mathrm{BiVO}_{4}$ film shows slightly higher absorption $(\sim 5-10 \%)$, but this difference is within the typical sample-to-sample variation of our spray pyrolysis technique. The resulting Tauc plot (Figure S3, Supplementary Information) shows that both films have nearly identical bandgap values. In summary, no significant optical or morphological changes are observed as a result of the introduction of $\mathrm{Ca}$ into $\mathrm{BiVO}_{4}$.
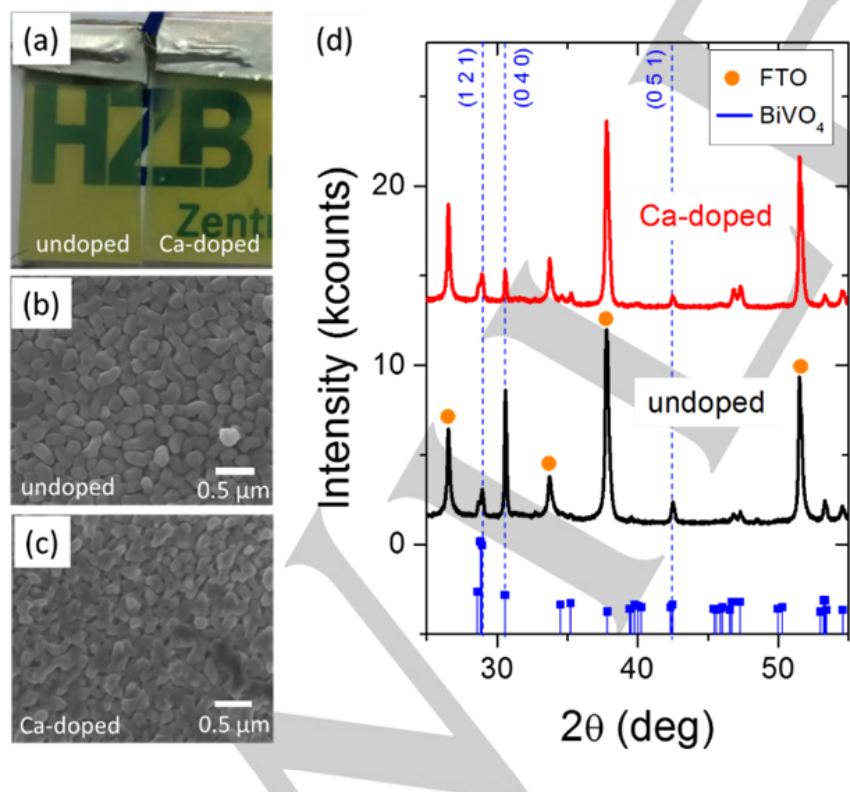

Figure 1. (a) Photograph of undoped and 1 at $\%$ Ca-doped $\mathrm{BiVO}_{4}$, deposited on FTO substrates. Scanning electron micrographs of (b) undoped and (c) 1 at\% Ca-doped $\mathrm{BiVO}_{4}$. (d) X-ray diffractograms of undoped and 1 at\% $\mathrm{Ca}$ doped $\mathrm{BiVO}_{4}$, showing the monoclinic scheelite structure.
Figure $1 \mathrm{~d}$ shows the X-ray diffractograms of undoped and Cadoped $\mathrm{BiVO}_{4}$ films. Other than peaks corresponding to the FTO substrates, only peaks of monoclinic $\mathrm{BiVO}_{4}$ are detected for both films. The intensity of the $(040)$ peak at $\sim 30.5^{\circ}$ is, however, reduced for the Ca-doped film. Moreover, this peak shifts towards lower $2 \theta$ values for increasing Ca content (see Fig. S4, Supplementary Information). This is a clear indication that $\mathrm{Ca}$ is successfully incorporated into the lattice, similar to what has been reported for e.g., $\mathrm{W}$-doped $\mathrm{BiVO}_{4}{ }^{[4 c]}$ The presence of $\mathrm{Ca}$ is also confirmed by X-ray photoelectron spectroscopy (XPS), as shown in Figure S5 (Supplementary Information). Finally, the Ca concentration in the Ca-doped film was quantified by inductively coupled plasma optical emission spectroscopy (ICP-OES); a value of $1.7 \pm 0.6$ at. \% was obtained, which is in reasonable agreement with the initial concentration of 1 at. \% $\mathrm{Ca}$ that was present in the precursor solution.

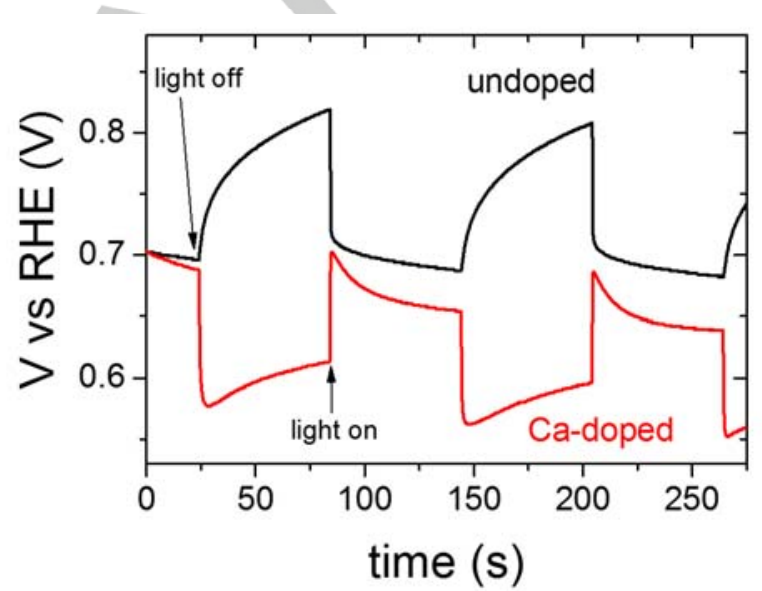

Figure 2. Open circuit potential of undoped and 1 at $\%$ Ca-doped $\mathrm{BiVO}_{4}$ under chopped AM1.5 illumination, measured in a $0.1 \mathrm{M}$ potassium phosphate buffer solution $\left(\mathrm{KP}_{\mathrm{i}}, \mathrm{pH} \sim 7\right)$.

To investigate the nature of the conductivity (n- or p-type) of the films, we measured the capacitance of the films to construct Mott-Schottky plots. However, obtaining reliable data was not possible since there were no conditions where the requirements for Mott-Schottky analysis are fulfilled for both films at all frequency range..$^{\text {[add }}$ ref] We therefore performed open circuit potential (OCP) measurements under chopped AM1.5 illumination, as shown in Figure 2. For the undoped $\mathrm{BiVO}_{4}$, the OCP shifts to a more negative value upon illumination. This is expected for an n-type semiconductor/electrolyte junction, where an upward bending of the energy bands (from the bulk to the surface) is present in the depletion layer. For the Ca-doped $\mathrm{BiVO}_{4}$, the opposite effect is observed; upon illumination, the OCP shifts instead to a more positive value. This indicates a downward band bending in the space charge region. This behavior is even more pronounced when the Ca concentration is increased to 10 at\% (Fig. S6, Supplementary Information). The change in sign of the OCP is a strong indication that the 
conductivity type of the $\mathrm{BiVO}_{4}$ has changed from n-type to p-type with the introduction of $\mathrm{Ca}$.

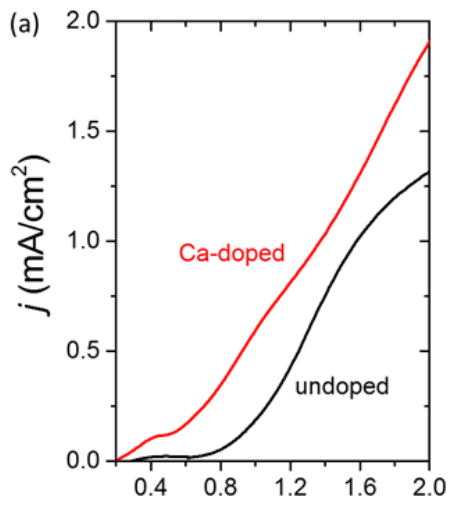

Potential vs RHE (V)

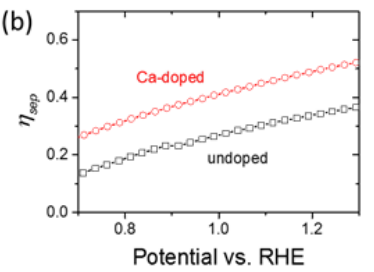

(c)

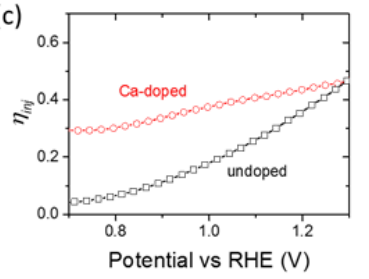

Figure 3. (a) AM1.5 photocurrent-potential curve of undoped and 1 at $\%$ Ca-doped $\mathrm{BiVO}_{4}$ photoelectrodes, measured in $0.1 \mathrm{M}$ potassium phosphate buffer solution $\left(\mathrm{KP}_{\mathrm{i}}, \mathrm{pH} \sim 7\right)$. By adding a hole scavenger $(0.5 \mathrm{M}$ $\mathrm{H}_{2} \mathrm{O}_{2}$ ) into the electrolyte solution and by measuring the optical absorption of the samples, it is possible to calculate (b) the carrier separation efficiency $\left(\eta_{\text {sep }}\right)$ and (c) carrier injection efficiency $\left(\eta_{\text {inj }}\right)$ of the samples as a function of applied potential. ${ }^{[7 \mathrm{~b}, 19]}$

Figure 3a shows the AM1.5 photocurrent-voltage curves of both the undoped and Ca-doped $\mathrm{BiVO}_{4}$ films, measured in a three-electrode configuration in a potassium phosphate buffer electrolyte $\left(0.1 \mathrm{M} \mathrm{KP_{i } , p H ~} \sim 7\right)$. A small oxidation peak was observed at 0.4-0.5 V vs. RHE for both films (although much smaller for the undoped film), which is attributed to partial oxidation of vanadium. ${ }^{[20]}$ Interestingly, despite the suggested p-type behavior from the OCP measurements, we did not observe any cathodic photocurrent (even down to $-0.2 \mathrm{~V}$ vs. $\mathrm{RHE}$ ) for the Ca-doped $\mathrm{BiVO}_{4}$. Instead, a significant ( 2-fold) increase of anodic photocurrent is observed after Ca-doping. To understand the cause of this significant photocurrent enhancement, we performed a hole scavenger study on both films, as previously reported in the literature. ${ }^{[\mathrm{b}, 19]}$ Hydrogen peroxide $\left(\mathrm{H}_{2} \mathrm{O}_{2}, 0.5 \mathrm{M}\right)$ is added into the $\mathrm{KP}_{\mathrm{i}}$ electrolyte and the AM1.5 photocurrent of the two samples was measured (see Fig. S7a, Supplementary Information). Based on this, the charge separation efficiency in the $\mathrm{BiVO}_{4}\left(\eta_{\text {sep }}\right)$ and the charge injection efficiency from the surface of $\mathrm{BiVO}_{4}$ into the electrolyte $\left(\eta_{\text {inj }}\right)$ can be calculated. ${ }^{[7 \mathrm{~b}, 19]}$ The results are shown in Figure $3 \mathrm{~b}$ and $3 \mathrm{c}$ for undoped and Ca-doped $\mathrm{BiVO}_{4}$. Based on these measurements, it is clear that $\mathrm{Ca}$ doping enhances both the carrier separation and injection efficiencies. The improvement is especially pronounced for the injection efficiency at low applied potentials. The product of the carrier separation and injection efficiencies for both films, which is equivalent to the internal quantum efficiency, is plotted along with the photocurrent-voltage curves, and shown in Fig. S7b (Supplementary Information). The very similar shape of the photocurrent curves and the $\eta_{\text {sep }} \times \eta_{\text {inj }}$ curves indicates that the photocurrent enhancement is indeed due to the enhanced charge separation and injection efficiencies. In other words, the improvement is not due to enhanced light absorption, which is consistent with the similar optical absorption spectra for undoped and Ca-doped $\mathrm{BiVO}_{4}$ in Fig. S2 (Supplementary Information). Incident photon-to-current conversion efficiencies (IPCE) of the undoped and Ca-doped $\mathrm{BiVO}_{4}$ films were also measured at $1.23 \mathrm{~V}$ vs. RHE under backside illumination in a phosphate buffer with added hole scavenger (Figure S8, Supplementary Information). Ca-doped film shows a factor of $\sim 2$ higher efficiency across the entire wavelength range. This is again consistent with the improvement observed under AM1.5 illumination (Fig.3 and Fig. S7).

To further reveal the nature behind the efficiency improvement, we performed a hard X-ray photoelectron spectroscopy (HAXPES) measurement on the Ca-doped $\mathrm{BiVO}_{4}$ film. The high photon energies allow the detection of photoelectrons that originated deeper within the bulk of the film. By varying the photon energy, we can therefore construct a compositional depth profile. Figure 4 shows the $\mathrm{Bi}$ $4 \mathrm{f}$ and $\mathrm{Ca} 2 \mathrm{p}$ spectra taken from the Ca-doped $\mathrm{BiVO}_{4}$ at varying photon energies. Three different photon energies were used: 2003, 4000 and $6009 \mathrm{eV}$. These energies correspond to information depths (defined as $3 \times$ the mean free path) of $\sim 8,13$, and $20 \mathrm{~nm}$, respectively. With increasing photon energy, the $\mathrm{Bi} 4 \mathrm{f}$ peak shows a systematic shift towards lower binding energies (see Fig. 4a and Supplementary Fig. S9). This means that the difference between the core level and the Fermi level at the surface is higher compared to that at the bulk. As shown in Supplementary Fig. S10, this implies a downward band bending, which agrees very well with the result of the OCP measurement.

There are two possible explanations for the observed downward shift in band bending in Ca-doped $\mathrm{BiVO}_{4}$. The first explanation is the presence of an accumulation layer in the (normally) n-type $\mathrm{BiVO}_{4}$. Such a layer could be formed when Ca occupies interstitial lattice sites and act as a donor-type dopant. However, in view of the large ionic radius of $\mathrm{Ca}^{2+}(>$ $100 \mathrm{pm}$ ), interstitial doping is highly unlikely. In addition, the total shift in binding energy is about $0.2 \mathrm{eV}$, which seems large for a potential drop in an accumulation layer. A second, more likely explanation is that $\mathrm{Ca}^{2+}$ substitutes for $\mathrm{Bi}^{3+}$ and acts as an acceptor-type dopant that induces a change from n-type to p-type conductivity according to Eq. (1). 
(a)

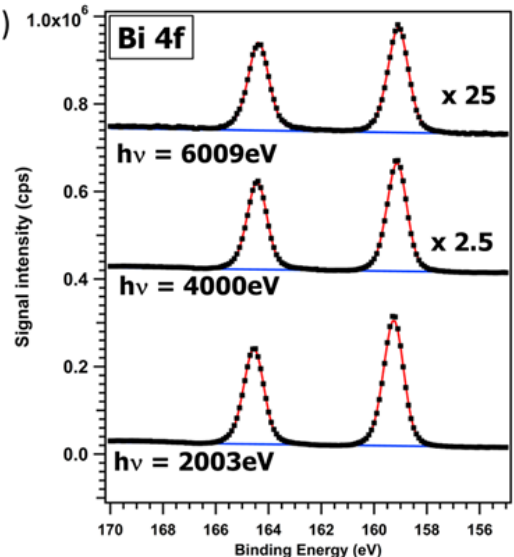

(b)

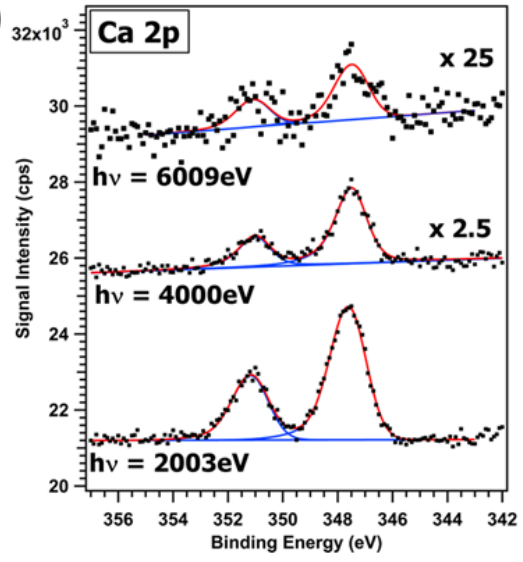

Figure 4. X-ray photoelectron spectra of 1 at $\% \mathrm{Ca}^{-\mathrm{BiVO}_{4}}$ sample, under varying $\mathrm{x}$-ray photon energies: 2003, 4000, and $6009 \mathrm{eV}$. The mean free path and the information depth of the measurement are increasing with increasing photon energy, i.e., we are increasingly bulk sensitive with increasing photon energy. Peaks associated with (a) Bi $4 \mathrm{f}$ and (b) $\mathrm{Ca} 2 \mathrm{p}$ core levels are shown. The symbols represent the measured data, and the lines represent the fitted curves.

Based on the $\mathrm{Ca} 2 \mathrm{p}$ and $\mathrm{Bi} 4 \mathrm{f}$ peaks (Fig. 4), the Ca:Bi ratio was calculated as a function of photon energy (i.e., depth in the film). The results, including the cross-section values for $\mathrm{Ca} 2 \mathrm{p}$ and $\mathrm{Bi} 4 \mathrm{f}$ peaks at different photon energies, are shown in Table 1. The $\mathrm{Ca}: \mathrm{Bi}$ ratio decreases from 0.11 (i.e., $\sim 5 \mathrm{at} \% \mathrm{Ca}$ ) measured at $2003 \mathrm{eV}$ excitation (more surface sensitive) to 0.033 (i.e., $\sim 1.5$ at\% Ca) measured at $6009 \mathrm{eV}$ excitation (more bulk sensitive). We have also attempted a cross-sectional energy dispersive X-ray (EDX) analysis to measure the $\mathrm{Ca}$ distribution, but a reliable analysis was not possible due to the overlapping peak position of $\mathrm{Ca}, \mathrm{Bi}$ and $\mathrm{Sn}$ and the relatively large sampling volume of the EDX technique $\left(0.1-5 \mathrm{\mu m}^{3}\right.$, depending on atomic number) compared to the film thickness. Nevertheless, it is clear from the HAXPES results (Table 1) that a gradient in $\mathrm{Ca}$ concentration is present in our Ca-doped film. Considering that $\mathrm{Ca}$ is initially distributed homogeneously in the spray precursor solution, this gradient in $\mathrm{Ca}$ concentration must form during the spray pyrolysis process and/or annealing treatment. The exact driving force for this out-diffusion is unknown. For example, Ca can out-diffuse from the bulk towards the surface of the $\mathrm{BiVO}_{4}$ film. Introduction of Ca may form islands of different phase on the surface that would promote the out-diffusion. However, this is rather unlikely, since there is no detectable phase in the X-ray diffractogram (Fig. 1d) and the XPS spectra of Ca shows no additional peak indicating phase segregation (Fig. $4 \mathrm{~b}$ ). Instead, we speculate that the chemical potential difference between the surface and the bulk (due to e.g., strain and surface energy) may result in Ca out-diffusion. ${ }^{[21]}$ Alternatively, self-diffusion (i.e., no driving force) process may also occur. Although this phenomenon has not yet been observed in $\mathrm{BiVO}_{4}$, selfdiffusion of $\mathrm{Ca}$ in other oxides (e.g., soda-lime silicate, calcite) has indeed been reported. ${ }^{[22]}$

Table 1. Cross-sections of $\mathrm{Ca} 2 \mathrm{p}$ and $\mathrm{Bi} 4 \mathrm{f}$ peaks for the photon energies used in our HAXPES measurement. The values are given in kilobarns $(\mathrm{kb})$, where $1 \mathrm{~kb}=10^{-25} \mathrm{~m}^{2}$. Based on these data and the measured peaks (Fig. $4)$, the $\mathrm{Ca}: \mathrm{Bi}$ ratios for different photon energies were calculated.

\begin{tabular}{llllll}
\hline $\begin{array}{l}\text { Photon } \\
\text { energy } \\
(\mathrm{eV})\end{array}$ & $\begin{array}{l}\mathrm{Ca} 2 \mathrm{p}_{3 / 2} \\
\text { cross- } \\
\text { section } \\
(\mathrm{kb})\end{array}$ & $\begin{array}{l}\mathrm{Ca} 2 \mathrm{p}_{1 / 2} \\
\text { cross- } \\
\text { section } \\
(\mathrm{kb})\end{array}$ & $\begin{array}{l}\mathrm{Bi} \\
\text { cross- } \\
\text { section } \\
(\mathrm{kb})\end{array}$ & $\begin{array}{l}\mathrm{Bi} \\
\text { cross- } \\
\text { section } \\
(\mathrm{kb})\end{array}$ & $\begin{array}{l}\mathrm{Ca}: \mathrm{Bi} \\
\text { ratio }\end{array}$ \\
\hline 2003 & 2.12 & 1.09 & 10.65 & 8.35 & 0.11 \\
4000 & 0.14 & 0.07 & 0.59 & 0.48 & 0.05 \\
6009 & 0.04 & 0.02 & 0.15 & 0.13 & 0.03 \\
\hline
\end{tabular}

Based on the results above, the following explanation for the observed efficiency enhancements in Ca-doped $\mathrm{BiVO}_{4}$ is proposed. In undoped n-type $\mathrm{BiVO}_{4}$, the Fermi level is constant throughout the thickness of the film and located close to the conduction band (Fig. 5a). This is, however, not the case for the Ca-doped $\mathrm{BiVO}_{4}$. Due to $\mathrm{Ca}$ out-diffusion, a gradient of concentration of $\mathrm{Ca}$ is present within the film. Since $\mathrm{Ca}$ is expected to be a shallow acceptor in $\mathrm{BiVO}_{4},{ }^{[14 a]}$ this will compensate the n-type conductivity of undoped $\mathrm{BiVO}_{4}$ (which presumably originates from intrinsic defects ${ }^{[23]}$ ). As a result, going from the bulk to the surface, the Fermi level will gradually shift closer towards the valence band (Fig. 5c). A gradual p-n homojunction is thus spontaneously formed within the Ca-doped $\mathrm{BiVO}_{4}$ film. The electric field within the homojunction is presumably the main reason for the enhanced carrier separation efficiency that we observed in Fig. $3 b$. 
(a) $\mathrm{BiVO}_{4}$

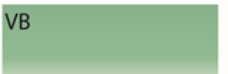

(c)

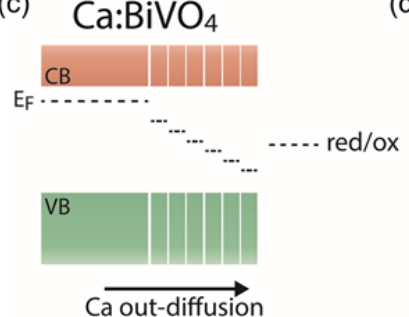

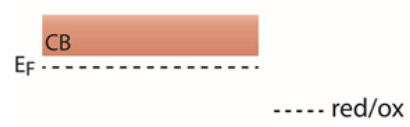

(b)
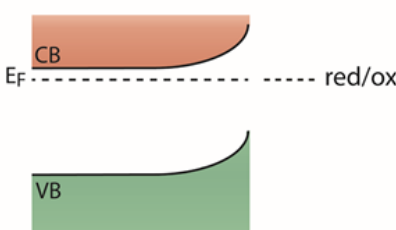

(d)

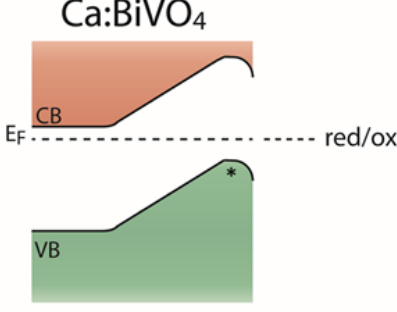

Figure 5. Band diagram of $(\mathbf{a}, \mathbf{b})$ undoped and $(\mathbf{c}, \mathbf{d}) \mathrm{Ca}$-doped $\mathrm{BiVO}_{4}$, before and after contact with redox electrolyte, respectively. CB: conduction band, VB: valence band, $\mathrm{E}_{\mathrm{F}}$ : Fermi level.

We now turn our attention to the surface region of the $\mathrm{BiVO}_{4}$. Upon contact with the electrolyte, the Fermi level of the $\mathrm{BiVO}_{4}$ will equilibrate with the redox level of the electrolyte. For undoped $\mathrm{BiVO}_{4}$, the Fermi level is located above the effective redox level of the electrolyte; this results in an upward band bending at the $\mathrm{BiVO}_{4} /$ electrolyte interface (Fig. 5b). In contrast, the Fermi level at the surface of Ca-doped $\mathrm{BiVO}_{4}$ is lower than the redox level. This, therefore, results in a downward band bending at the $\mathrm{BiVO}_{4} /$ electrolyte interface (Fig. $5 \mathrm{~d}$ ). The downward band bending is fully consistent with the OCP and HAXPES measurements discussed above.

We note that by itself, the downward band bending and p-type behavior at the surface seem inconsistent with the observation of anodic photocurrents in Fig. 3a. The anodic photocurrents can however, be understood when also taking into account the $p$ type region in the bulk of the film (Fig. $5 \mathrm{~d}$ ). The holes that are photo-generated in the bulk of the film will travel toward the surface and will accumulate in the region marked with the asterisk. Since the concentration of electrons is very low in this p-type region, no recombination occurs and the concentration of holes will continue to increase. As a result, the bands at the surface will flatten, after which the holes can be injected into the electrolyte. The p-n junction model in Fig. $5 d$ also explains why no cathodic photocurrents are observed.

\section{Conclusions}

In conclusion, Ca-doped $\mathrm{BiVO}_{4}$ was successfully prepared via spray pyrolysis. The change in OCP under illumination reflects behavior that is typical for a p-type semiconductor, which indicates that $\mathrm{Ca}$ indeed acts as an acceptor-type dopant in
$\mathrm{BiVO}_{4}$. However, no cathodic photocurrent is observed; instead, an enhancement of the anodic photocurrent is found. Through hole scavenger and HAXPES measurements, we found that the enhancement is caused by Ca out-diffusion towards the surface of the film, which results in the spontaneous formation of a gradual $\mathrm{p}-\mathrm{n}$ homojunction within the $\mathrm{BiVO}_{4}$ film. The built-in electric field enhances the carrier separation efficiency, while the p-n junction explains the absence of a cathodic photocurrent. In addition, an increase in the carrier injection efficiency is observed, which is tentatively attributed to a higher catalytic activity of Ca-rich $\mathrm{BiVO}_{4}$ surfaces for water oxidation. Overall, this study demonstrates the sometimes surprising and unexpected role(s) that dopants can play in metal oxide photoelectrodes. As illustrated here for spontaneously formed $\mathrm{p}$ $\mathrm{n}$ homojunctions in Ca-doped $\mathrm{BiVO}_{4}$, controlled doping may not only improve the performance, but may also enable new device architectures for known photoelectrode materials.

\section{Experimental Section}

\section{Synthesis}

Thin films of $\mathrm{BiVO}_{4}$ were prepared using spray pyrolysis. The preparation of the precursor solution and the spray pyrolysis setup are described elsewhere. ${ }^{[7 \mathrm{~b}, 17 \mathrm{~b}]}$ For the preparation of Ca-doped $\mathrm{BiVO}_{4}$, varying amounts of $\mathrm{Ca}\left(\mathrm{NO}_{3}\right)_{2} \cdot 4 \mathrm{H}_{2} \mathrm{O}$ (puriss. p.a., ACS reagent, $99-103 \%$, Sigma Aldrich) were added to the $\mathrm{BiVO}_{4}$ precursor solution. The ratio of $(\mathrm{Ca}+\mathrm{Bi})$ to $\mathrm{V}$ in the mixture is kept at $1: 1$. The spray deposition for both undoped and $\mathrm{Ca}$-doped $\mathrm{BiVO}_{4}$ was done for 100 cycles, with a spray deposition rate of $\sim 1 \mathrm{~nm}$ per cycle. As substrates, either FTO-coated glass $(2 \times 2$ $\mathrm{cm}$, TEC-15, Hartford Glass Co.) or fused silica ('quartz', $2.5 \times 1.2 \mathrm{~cm}$, Spectrosil ${ }^{\circledR} 2000$, Heraeus) were used. In contrast to our previous reports, ${ }^{[17,24]}$ no $\mathrm{SnO}_{2}$ layer was deposited between the FTO substrate and the $\mathrm{BiVO}_{4}$ film. After deposition, all samples were annealed for $2 \mathrm{~h}$ at $450^{\circ} \mathrm{C}$ in air to further improve the crystallinity.

\section{Photoelectrochemical measurements}

Photoelectrochemical measurements were done in an aqueous $0.1 \mathrm{M}$ potassium phosphate buffer electrolyte solution $(\mathrm{pH} \sim 7)$ in a threeelectrode configuration. The potential of the working electrode was controlled by a potentiostat (EG\&G PAR 273A). A Pt wire and an $\mathrm{Ag} / \mathrm{AgCl}$ electrode (XR300, saturated $\mathrm{KCl}$ and $\mathrm{AgCl}$ solution, Radiometer Analytical) were used as the counter and reference electrodes, respectively. Cyclic voltammetry measurements were performed with a scan rate of $50 \mathrm{mV} / \mathrm{s}$. White light photocurrent measurements were performed under simulated AM1.5 solar illumination $\left(100 \mathrm{~mW} / \mathrm{cm}^{2}\right)$ with a WACOM Super Solar Simulator (Model WXS-505-5H). A home-built Teflon photoelectrochemical cell was used with a $6 \mathrm{~mm}$ diameter opening for sample exposure to the electrolyte. Open circuit potential measurements were done using the same configuration by setting the potentiostat to the "open cell" condition. Hole scavenger measurements were done by adding $0.5 \mathrm{M}$ hydrogen peroxide (30\%, Merck Schuchardt $\mathrm{OHG}$ ) into the phosphate buffer electrolyte. Incident photon-to-current conversion efficiencies (IPCE) measurements utilized a $300 \mathrm{~W}$ xenon lamp (Oriel) connected with a grating monochromator (Acton Spectra Pro 2155).

\section{$\underline{\text { Characterization }}$}


X-ray diffraction (XRD) measurements were performed on the $\mathrm{BiVO}_{4}$ thin films using a Bruker D8 Advance diffractometer with $\mathrm{Cu} \mathrm{K} \alpha$ radiation at $40 \mathrm{kV}$ and $40 \mathrm{~mA}$. Scanning electron microscopy (SEM) images were obtained using a LEO GEMINI 1530. UV-vis measurements were performed on the $\mathrm{BiVO}_{4}$ films deposited on quartz using a PerkinElmer Lambda 950 spectrophotometer with an integrating sphere. The films were placed inside the integrating sphere using a center mount sample holder to measure transflectance (i.e., transmittance + reflectance). The transflectance of the bare quartz substrate was used as the $100 \%$ transflectance baseline for the $\mathrm{BiVO}_{4}$ films. The absorptance of the $\mathrm{BiVO}_{4}$ films was calculated as follows: absorptance $=1-$ transflectance. Inductively coupled plasma optical emission spectroscopy (ICP-OES) was performed using iCAP 7000 series spectrometer (Thermo Scientific). Ca-doped $\mathrm{BiVO}_{4}$ film was fully dissolved in $1 \mathrm{M}$ hydrochloric acid $(\mathrm{HCl})$, and the solution was fed into the spectrometer. Lab-based X-ray photoelectron spectroscopy (XPS) was carried out with a monochromatic Al Ka X-ray source (1486.74 eV, Specs Focus 500 monochromator) and a hemispherical analyzer (Specs Phoibos 100) in an ultrahigh vacuum system (base pressure $\sim 10^{-8} \mathrm{mbar}$ ). Hard X-ray photoelectron spectroscopy (HAXPES) measurements were carried out at BESSY II synchrotron facility (HiKE end station, KMC-1 beamline, ${ }^{[25]}$ HelmholtzZentrum Berlin, Germany), where a photon excitation energy in the range from 2000 to $12000 \mathrm{eV}$ is available. Three different fixed photon energies were used in our measurements: 2003, 4000 and $6009 \mathrm{eV}$ (first order diffracted light from the $\mathrm{Si}(111), \mathrm{Si}(311)$, and $\mathrm{Si}(422)$ planes of the double-crystal monochromator, respectively). The analyzer was a Scienta R4000 optimized for high kinetic energies up to $10000 \mathrm{eV}$. The binding energy scale was calibrated with the Au Fermi edge $\left(E_{F}\right)$ measured from a gold foil placed on the side of the sample. During the measurements, the pressure was around $10^{-8}$ mbar in the analysis chamber

\section{Acknowledgements}

We acknowledge Christian Höhn for his assistance with the XPS measurements as well as Alexander Esau and Peter Bogdanoff for their help with the ICP-OES measurement. Part of this work was funded by Europe's Fuel Cell and Hydrogen Joint Undertaking (PECDEMO project, Grant Agreement no. 621252).

Keywords: solar water splitting $\cdot$ photoelectrode $\cdot \mathrm{BiVO}_{4} \cdot$ doping $\cdot p-n$ junction

[1] aK. Sivula, The Journal of Physical Chemistry Letters 2013, 4, 1624-1633; bC. Jiang, S. J. A. Moniz, A. Wang, T. Zhang, J. Tang, Chem. Soc. Rev. 2017, 46, 46454660; cY. Yang, S. Niu, D. Han, T. Liu, G. Wang, Y. Li, Adv. Energy. Mater. 2017, 7, 1700555.

[2] aY. Park, K. J. McDonald, K. S. Choi, Chem. Soc. Rev. 2013, 42, 2321-2337; bH.

L. Tan, R. Amal, Y. H. Ng, Journal of Materials Chemistry A 2017, 5, 1649816521; cF. F. Abdi, S. P. Berglund, J. Phys. D: Appl. Phys. 2017, 50, 193002.
[3] aC. Zachäus, F. F. Abdi, L. M. Peter, R. van de Krol, Chemical Science 2017, 8, 3712-3719; bY. Ma, A. Kafizas, S. R. Pendlebury, F. Le Formal, J. R. Durrant, Adv. Funct. Mater. 2016; cY. Ma, F. Le Formal, A. Kafizas, S. R. Pendlebury, J. R. Durrant, Journal of Materials Chemistry A 2015, 3, 20649-20657.

[4] aS. K. Choi, W. Choi, H. Park, Phys. Chem. Chem. Phys. 2013; bJ. A. Seabold, K. S. Choi, J.Am.Chem.Soc. 2012, 134, 2186-2192; cD. K. Zhong, S. Choi, D. R. Gamelin, J. Am. Chem. Soc. 2011, 133, 18370-18377; dB. Lamm, B. J.

Trześniewski, H. Döscher, W. A. Smith, M. Stefik, ACS Energy Lett. 2017, $112-$ 124; eY. Tang, R. Wang, Y. Yang, D. Yan, $\mathrm{X}$. Xiang, ACS Appl. Mater. Interf. 2016, 8, 19446-19455.

[5] aF. F. Abdi, T. J. Savenije, M. M. May, B. Dam, R. van de Krol, The Journal of Physical Chemistry Letters 2013, 4, 27522757; bM. Ziwritsch, S. n. Müller, H. Hempel, T. Unold, F. F. Abdi, R. van de Krol, D. Friedrich, R. Eichberger, ACS Energy Lett. 2016, 1, 888-894.

[6] A. J. E. Rettie, H. C. Lee, L. G. Marshall, J.-F. Lin, C. Capan, J. Lindemuth, J. S. McCloy, J. Zhou, A. J. Bard, C. B. Mullins, Journal of the American Chemical Society 2013, 135, 11389-11396.

[7] aK. P. S. Parmar, H. J. Kang, A. Bist, P. Dua, J. S. Jang, J. S. Lee, ChemSusChem 2012, 5, 1926-1934; bF. F. Abdi, N. Firet, R. van de Krol, ChemCatChem 2013, 5 , 490-496.

[8] aX. Shi, K. Zhang, K. Shin, M. Ma, J. Kwon, I. T. Choi, J. K. Kim, H. K. Kim, D. H. Wang, J. H. Park, Nano Energy 2015, 13, 182-191; bX. Shi, I. Y. Choi, K. Zhang, J. Kwon, D. Y. Kim, J. K. Lee, S. H. Oh, J. K. Kim, J. H. Park, Nat. Commun. 2014, 5; cL. Chen, F. M. Toma, J. K. Cooper, A. Lyon, Y. Lin, I. D. Sharp, J. W. Ager, ChemSusChem 2015; dF. F. Abdi, L. Han, A. H. M. Smets, M. Zeman, B. Dam, R. van de Krol, Nat. Commun. 2013, 4. 
[9] aH. He, S. P. Berglund, A. J. E. Rettie, W. D. Chemelewski, P. Xiao, Y. Zhang, C. B. Mullins, Journal of Materials Chemistry A 2014, 2, 9371-9379; bX. Shi, H. Jeong, S. J. Oh, M. Ma, K. Zhang, J. Kwon, I. T. Choi, I. Y. Choi, H. K. Kim, J. K. Kim, Nat. Commun. 2016, 7.

[10] R. Liu, J. Ren, D. Zhao, J. Ning, Z. Zhang, Y. Wang, Y. Zhong, C. Zheng, Y. Hu, Inorganic Chemistry Frontiers 2017, 4, 2045-2054.

[11] J. H. Kim, Y. Jo, J. H. Kim, J. W. Jang, H. J. Kang, Y. H. Lee, D. S. Kim, Y. Jun, J. S. Lee, ACS nano 2015, 9, 11820-11829.

[12] Z. Jiao, J. Zheng, C. Feng, Z. Wang, X. Wang, G. Lu, Y. Bi, ChemSusChem 2016, 9, 2824-2831.

[13] aC. Liu, X. Li, J. Su, L. Guo, Int. J. Hydrogen Energy 2016; bF. Wang, W. Septina, A. Chemseddine, F. F. Abdi, D. Friedrich, P. Bogdanoff, R. van de Krol, S. D. Tilley, S. P. Berglund, J. Am. Chem. Soc. 2017, 139, 15094-15103.

[14] aW. J. Yin, S. H. Wei, M. M. Al-Jassim, J. Turner, Y. Yan, Phys. Rev. B 2011, 83; bK. Ding, L. Wen, L. Xu, H. Wu, Y. Ye, Y. Zhang, Int. J. Quantum Chem 2016, 116, 388-395.

[15] Y.-M. Chiang, D. Birnie III, D. W. Kingery, Physical Ceramics, Wiley, New York, 1997.

[16] aW. F. Yao, J. H. Ye, J. Phys. Chem. B 2006, 110, 11188-11195; bS. F. Sameera, P. P. Rao, L. S. Kumari, P. Koshy, Chem. Lett. 2009, 38, 1088-1089; cD. Yaomin, B. Jianjiang, Ceram. Int. 2013, 39, 1555-1560.

[17] aF. F. Abdi, R. van de Krol, J. Phys. Chem. C 2012, 116, 9398-9404; bF. F. Abdi, N. Firet, A. Dabirian, R. van de Krol, MRS Online Proceedings Library 2012, 1446, null-null.

[18] aZ. Xiang, Y. Wang, D. Zhang, P. Ju, Journal of Industrial and Engineering Chemistry 2016; bM. C. Long, W. M. Cai, H. Kisch, Journal of Physical Chemistry C 2008, 112, 548-554; cM. Long, W. Cai, J. Cai, B. Zhou, X. Chai, Y. Wu, J. Phys.
Chem. B 2006, 110, 20211-20216; dX. Zou, Y. Dong, X. Zhang, Y. Cui, X. Ou, X. Qi, Appl. Surf. Sci. 2016.

[19] H. Dotan, K. Sivula, M. Gratzel, A. Rothschild, S. C. Warren, Energy Environ. Sci. 2011, 4, 958-964.

[20] aB. J. Trześniewski, W. A. Smith, Journal of Materials Chemistry A 2016, 4, 29192926; bHandbook of Chemistry and Physics, 72 ed., CRC Press, Boca Raton, 1992.

[21] C.-Y. Hui, A. Jagota, Langmuir 2013, 29, 11310-11316.

[22] aH. Mehrer, A. W. Imre, E. Tanguep-

Nijokep, in Journal of Physics:

Conference Series, Vol. 106, IOP

Publishing, 2008, p. 012001; bD. K. Fisler, R. T. Cygan, Am. Mineral. 1999, 84, 13921399.

[23] J. K. Cooper, S. B. Scott, Y. Ling, J. Yang, S. Hao, Y. Li, F. M. Toma, M. Stutzmann, K. V. Lakshmi, I. D. Sharp, Chem. Mater. 2016, 28, 5761-5771.

[24] Y. Q. Liang, T. Tsubota, L. P. A. Mooij, R. Van de Krol, Journal of Physical Chemistry C 2011, 115, 17594-17598.

[25] F. Schaefers, M. Mertin, M. Gorgoi, Rev. Sci. Instrum. 2007, 78, 123102. 


\section{Entry for the Table of Contents}

\section{FULL PAPER}

\section{Dopant diffusion: Poor carrier} separation represents one of the major limitations in the development of metal oxide photoelectrodes. Introducing $\mathrm{Ca}$ as an acceptor-type dopant in $\mathrm{BiVO}_{4}$ photoelectrodes spontaneously creates a $p-n$ junction in the film due to $\mathrm{Ca}$ out-diffusion. As a result, the charge carrier separation is enhanced, and the AM1.5 photocurrent is improved by a factor of two.

\section{Ca-doped $\mathrm{BiVO}_{4}$}

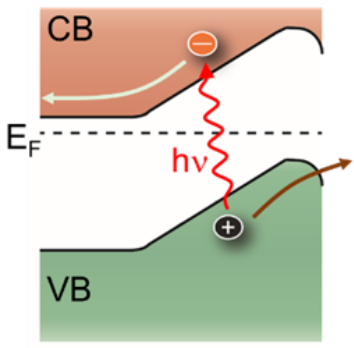

Ca out-diffusion
F. F. Abdi*, D. E. Starr, I.Y. Ahmet, R. van de Krol

\section{Page No. - Page No.}

Photocurrent enhancement by spontaneous formation of a p-n junction in Ca-doped bismuth vanadate photoelectrodes 